\title{
Procréer en temps d'infection à VIH : offre de soins et expériences de femmes en milieu urbain (Burkina Faso)
}

Thèse de Doctorat (PhD) en anthropologie, Université d'Aix-Marseille (France), 2012

Sylvie Zongo

\section{OpenEdition Journals}

Édition électronique

URL : http://journals.openedition.org/anthropodev/388

DOI : 10.4000/anthropodev.388

ISSN : 2553-1719

Éditeur

APAD - Association pour l'anthropologie du changement social et du développement

\section{Édition imprimée}

Date de publication : 1 septembre 2015

Pagination : 251-252

ISBN : 979-10-93476-02-5

ISSN : 2276-2019

Référence électronique

Sylvie Zongo, «Procréer en temps d'infection à VIH : offre de soins et expériences de femmes en milieu urbain (Burkina Faso)», Anthropologie \& développement [En ligne], 42-43 | 2015, mis en ligne le 01 décembre 2016, consulté le 24 septembre 2020. URL : http://journals.openedition.org/ anthropodev/388; DOI : https://doi.org/10.4000/anthropodev.388

\section{(c) ()}

La revue Anthropologie \& développement est mise à disposition selon les termes de la Licence Creative Commons Attribution 4.0 International. 


\title{
Procréer en temps d'infection à VIH: offre de soins et expériences de femmes en milieu urbain (Burkina Faso)
}

\author{
Sylvie Zongo ${ }^{1}$ \\ Thèse de Doctorat (PhD) en anthropologie, \\ Université d'Aix-Marseille (France), 2012
}

\section{Résumé}

Cette thèse porte sur la procréation dans le contexte de l'infection à VIH marqué par des progrès thérapeutiques engrangés dans le traitement de l'infection. Elle est construite autour du questionnement suivant: "à quelles conditions (sociales, sanitaires, économiques, etc.) devient-on mère pour une femme porteuse du VIH au Burkina Faso? " Nous nous intéressons d'une part à la chronicisation de l'infection à $\mathrm{VIH}$ et à ses effets sur les individus et leurs projets procréatifs et, d'autre part, à l'impact de cette chronicisation sur les systèmes de santé et les associations de lutte contre le VIH, et précisément sur leurs capacités à accompagner les individus dans leurs projets.

Du fait de sa transmission sexuelle et de la mère à l'enfant, l'infection à VIH a longtemps été considérée comme une barrière sérieuse à la reproduction. Les désirs de procréation des individus séropositifs étaient parfois réprimés ou se heurtaient dans bien des cas à des systèmes de santé peu aptes à prendre en charge ces personnes. L'accès aux traitements ARV et la mise au point de protocoles thérapeutiques permettant de réduire la transmission du virus à l'enfant, tout en ouvrant la voie vers la parentalité, ont fait émerger des problématiques nouvelles tant pour les individus concernés que pour les acteurs qui interviennent dans la prise en charge du VIH (associations, professionnels de la santé, institutions étatiques ou de coopération, etc.).

L'un des postulats qui a structuré notre thèse est que la procréation dans le contexte du VIH n'est pas réductible au désir d'enfant et ne constitue pas un ensemble homogène, même si les femmes sont liées par l'infection à VIH et que le fait d'avoir un enfant reste in fine important dans la construction de leur féminité. Les configurations sont diverses, les priorités ne sont pas les mêmes du fait des situations conjugales, sérologiques et économiques des unes et des autres. Ceci induit, pour les femmes, des choix et des parcours de soins plus ou moins différenciés que

${ }^{1}$ E-mail : zongosyl@yahoo.fr 
les agences, les acteurs associatifs, les professionnels de la santé influencent fortement suivant une logique de prévention des risques sanitaires.

Cette logique sanitaire est apparue tout au long du contact avec le terrain comme l'élément structurant de l'accès à la parentalité. En effet, la sexualité (reproductive), la grossesse, l'accouchement, la maternité (suivi post-natal de l'enfant jusqu'au dépistage) sont de plus en plus médicalisés, au point que, d'une part, ne pas bénéficier d'un accompagnement médical au cours de ces étapes est interprété comme un écart et, d'autre part, parler de la procréation en situation de séropositivité c'est se heurter à la reproduction de discours médicalisés et orientés vers la prévention des risques sanitaires. Les professionnels de la santé et aussi les associations se posent en censeurs de la légitimité du projet reproductif et du parcours génésique des femmes.

Les stratégies de contournement des normes médicales, surtout dans la phase de la quête de grossesse, témoignent des capacités dont elles disposent à se soustraire du contrôle médical de leur vie reproductive. Ces capacités, elles les développent aussi dans la gestion de leur maternité dans la séropositivité, où les constructions de l'enfant, la relation à l'enfant, les stratégies développées pour ne pas contaminer l'enfant permettent de relativiser leur "vulnérabilité " et de leur reconnaître ainsi des capacités d'actions individuelles. 